\title{
An Iterative Finite-Difference Method for Hyperbolic Systems
}

\author{
By S. Abarbanel and G. Zwas
}

\begin{abstract}
An iterative finite-difference scheme for initial value problems is presented. It is applied to the quasi-linear hyperbolic system representing the one-dimensional time dependent flow of a compressible polytropic gas. The emphasis in this research was on the handling of discontinuities, such as shock waves, and overcoming the post-shock oscillations resulting from nonlinear instabilities. The linear stability is investigated as well. The success of the method is indicated by the monotonic profiles which were obtained for almost all the cases tested.
\end{abstract}

1. Introduction. In this paper we describe a finite-difference scheme of an iterative character which is used to solve initial value problems. In particular, we examine quasi-linear hyperbolic systems, such as the one describing the onedimensional time dependent flow of a compressible polytropic gas. The emphasis in this research was on the handling of discontinuities, such as shock waves, and overcoming the post-shock oscillations resulting from nonlinear instabilities. The success of the method is indicated by the monotonic profiles which were obtained for almost all the cases tested.

The method is based on an idea which was first examined by Gary [1] and considered by him to be unsatisfactory [2]. It will be shown what modifications are needed to insure stability and monotonicity of the flow property profiles. Recently Gourlay and Morris [9] used a similar approach to deal with problems which have smooth solutions.

The scheme is compared with several others, including the one proposed by Godunov, by performing the numerical computations for two hydrodynamic problems:

(1) Plane, steady shock wave.

(2) A plane detonation wave reflecting off a wall.

The computations described herein were carried out on the CDC 3400 at Tel-Aviv University.

2. The Differential Equations. The system of partial differential equations under consideration is

$$
\partial W / \partial t+A \cdot \partial W / \partial x=0 .
$$

The independent variables are $x$ and $t ; W$ is the vector of the unknown (scalar) functions $\left(W_{1}, W_{2}, \cdots, W_{n}\right)$ and $A$ is a matrix whose components are functions of the $W$ 's. The system is quasi-linear because $A$ does not contain derivatives of $W$. The hyperbolicity of the equation implies that the eigenvalues of $A$, i.e. $\lambda_{1}\left(W_{1}, \cdots, W_{n}\right), \cdots, \lambda_{n}\left(W_{1}, \cdots, W_{n}\right)$, are real for all values of $W_{1}, \cdots, W_{n}$ under consideration.

Received May 6, 1968, revised November 7, 1968. 
Equation (1) is said to be in a conservation-law form if $A(\partial W / \partial x)=\partial F / \partial x$ i.e.

$$
\partial W / \partial t=-\partial F / \partial x
$$

where $F$ is a vector whose components are functions of the components of $W$. In this paper all our numerical computations and stability analysis will be carried out for the one-dimensional hydrodynamic equations, in Lagrangian coordinates, for a polytropic gas. For this case we have [3]

$$
W=\left[\begin{array}{c}
v \\
u \\
E
\end{array}\right] ; \quad F(W)=\left[\begin{array}{c}
-u \\
p \\
p u
\end{array}\right]
$$

where $V, u, E$ and $p$ are respectively the specific volume, velocity, specific total energy and the pressure.* All quantities are dimensionless, including the time $t$ and the Lagrangian coordinate $x$. The corresponding form of $A$ is

$$
A(\mathrm{~W})=-\frac{1}{V}\left[\begin{array}{ccc}
0 & -V & 0 \\
-p & -(\gamma-1) u & \gamma-1 \\
-p u & p V-(\gamma-1) u^{2} & (\gamma-1) u
\end{array}\right] .
$$

3. The Difference Equations. As the finite-difference approximation to (1a) we chose the Lax-Wendroff scheme [4], [3] which is of second-order accuracy:

$$
\begin{aligned}
W_{j}^{n+1}= & W_{j}^{n}-\frac{\lambda}{2}\left[F_{j+1}^{n}-F_{j-1}^{n}\right] \\
& +\frac{\lambda^{2}}{2}\left[A_{j+1 / 2}^{n}\left(F_{j+1}^{n}-F_{j}{ }^{n}\right)-A_{j-1 / 2}^{n}\left(F_{j}{ }^{n}-F_{j-1}^{n}\right)\right]
\end{aligned}
$$

where $f_{j}{ }^{n}=f\left(x_{j}, t_{n}\right)$ and $\lambda=\Delta t / \Delta x$. Equation (4) may be written as

$$
W_{j}^{n+1}=W_{j}^{n}+Q \cdot W_{j}^{n} .
$$

For the treatment of shock waves Lax and Wendroff [4] suggest adding to Eq. (5) another term $Q^{\prime} \cdot W_{j}^{n}$ which plays the role of artificial viscosity. The equation then takes the form

$$
W_{j}^{n+1}=\left[I+Q+Q^{\prime}\right] W_{j}^{n}
$$

with

$$
\begin{aligned}
Q^{\prime} \cdot W_{j}^{n}=\frac{a \lambda}{2}\left[\frac{c_{j+1}^{n}-c_{j}^{n}}{\left(c_{j+1 / 2}^{n}\right)^{2}}\left(A_{j+1 / 2}^{n}\right)^{2}\left(W_{j+1}^{n}-W_{j}^{n}\right)\right. \\
\\
\left.\quad-\frac{c_{j}^{n}-c_{j-1}^{n}}{\left(c_{j-1 / 2}^{n}\right)^{2}}\left(A_{j-1 / 2}^{n}\right)^{2}\left(W_{j}^{n}-W_{j-1}^{n}\right)\right]
\end{aligned}
$$

where $c$ is the Lagrangian sound speed $\left(=(\gamma p / v)^{1 / 2}\right)$ and $a$ is a constant of order unity. A linear stability analysis, à la von Neumann, yields the following stability criterion [3]:

* Here the pressure is given by $p=((\gamma-1) / v)\left[E-\frac{1}{2} u^{2}\right]$, where $\gamma$ is the polytropic constant. 


$$
\frac{\Delta t}{\Delta x}=\lambda \leqq \frac{\left(1+a^{2} / 4\right)^{1 / 2}-a / 2}{\operatorname{Max}_{j}\left|c_{j}{ }^{n}\right|} .
$$

This follows from the fact that $c$ (the Lagrangian sound speed) is the maximal characteristic value of $A$. Thus $a=3 / 2$ gives a requirement which is twice as stringent as the usual CFL (Courant-Friedrichs-Levy) criterion. In the present work, however, we shall use mainly schemes that stem from Eq. (5) rather than (6).

We have not specified as yet how one defines $A_{j+1 / 2}^{n}$. In the open literature one finds two versions [1], [3], [5], [4]:

$A_{j+1 / 2}^{n}=\frac{1}{2}\left[A_{j+1}^{n}+A_{j}^{n}\right]=\frac{1}{2}\left[A\left(W_{j+1}^{n}\right)+A\left(W_{j}^{n}\right)\right]$ and $A_{j+1 / 2}^{n}=A\left[\left(W_{j+1}^{n}+W_{j}^{n}\right) / 2\right]$.

Both forms preserve the integrated conservation law, but in general the resulting difference equation does not satisfy the conservation requirements "in the small." In other words, usually $A_{j+1 / 2} \cdot \Delta W \neq \Delta F$. However, the L-W method is based on obtaining the second term in a Taylor series thus:

$$
W_{t t}=\left(-F_{x}\right)_{t}=\left(-F_{t}\right)_{x}=\left(-A W_{t}\right)_{x}=\left(A F_{x}\right)_{x}
$$

where the third equality is due to the fact that $A$ is the Jacobian of $F$ with respect to $W$. The equivalent finite-difference relation would be $A \cdot \Delta w=\Delta F$. This requirement is satisfied if one takes $A_{j+1 / 2}^{n}=A\left[\left(W_{j+1}^{n}+W_{j}^{n}\right) / 2\right]$ where $A(W)$ is given by (3) with $p$ appearing explicitly as shown. If, for example, one takes $A_{j+1 / 2}^{n}=$ $A\left[\left(W_{j+1}^{n}+W_{j}{ }^{n}\right) / 2\right]$ where, however, $A(W)$ is given in terms of the proper components of $W$, i.e. $v, u$ and $E$, then $A \cdot \Delta W$ is not exactly equal to $\Delta F$.

It turns out that in practical computations the numerical results obtained by our iterative scheme are very similar for all versions of $A_{j+1 / 2}^{n}$. We feel, however, that in multi-dimensional problems, an analogous truly conservative scheme might be the more successful one.

We close this section by noting that

$$
A_{j+1 / 2}^{n} \cdot \Delta w=\Delta F
$$

implies

$$
\left(A_{j+1 / 2}^{n}\right)^{2} \cdot \Delta W=A_{j+1 / 2}^{n} \cdot \Delta F .
$$

Note that using (9) one may obtain (see Eq. (6))

$$
\begin{aligned}
\left(Q+Q^{\prime}\right) W_{j}^{n}=-\frac{\lambda}{2}\left(F_{j+1}^{n}-F_{j-1}^{n}\right) & \cdot\left\{\left[1+\frac{a}{\lambda} \frac{\left(c_{j+1}^{n}-c_{j}^{n}\right)}{\left(c_{j+1 / 2}^{n}\right)^{2}}\right] A_{j+1 / 2}^{n}\left(F_{j+1}^{n}-F_{j}{ }^{n}\right)\right. \\
(10) \quad & {\left.\left[1+\frac{a}{\lambda} \frac{\left(c_{j}^{n}-c_{j-1}^{n}\right)}{\left(c_{j-1 / 2}^{n}\right)^{2}}\right] A_{j-1 / 2}^{n}\left(F_{j}^{n}-F_{j-1}^{n}\right)\right\} . }
\end{aligned}
$$

We thus see that the effect of adding the artificial viscosity term merely changes the coefficients of the terms of the form $A \cdot \Delta F$. It is for this reason that the iterative procedure, to be described shortly, was applied to Eq. (5) rather than to (6).

We conclude by noting that 


$$
\begin{aligned}
A_{j+1 / 2}^{n} \cdot\left(F_{j+1}^{n}-F_{j}\right) & =\left(A_{j+1 / 2}^{n}\right)^{2}\left(W_{j+1}^{n}-W_{j}^{n}\right) \\
& =\frac{1}{2}\left[\begin{array}{c}
-2\left(p_{j+1}^{n}-p_{j}^{n}\right) \\
2 \gamma \frac{p_{j+1}^{n}+p_{j}^{n}}{v_{j+1}^{n}+v_{j}^{n}\left(u_{j+1}^{n}-u_{j}^{n}\right)} \\
\gamma \frac{p_{j+1}^{n}+p_{j}^{n}}{v_{j+1}^{n}+v_{j}^{n}}\left[\left(u_{j+1}^{n}\right)^{2}-\left(u_{j}^{n}\right)^{2}\right]+\left(p_{j+1}^{n}\right)^{2}-\left(p_{j}^{n}\right)^{2}
\end{array}\right]
\end{aligned}
$$

and similarly for $A_{j-1 / 2}^{n} \cdot\left(F_{j}^{n}-F_{j-1}^{n}\right)$. This result is similar but not equal to the one quoted by Lax and Wendroff [3] since $\bar{c}^{2} \neq \gamma \bar{p} / \bar{v}$. We feel that the right-hand side of (11) is in the more proper form because of the equality of the left-hand side and middle terms. This equality is assured by choosing $A_{j+1 / 2}^{n}$ as described above.

4. The Iterative Method. This is an explicit-implicit scheme in which the solution of the difference equations is taken as the zeroth approximation. Write the difference system in the fully explicit form

$$
W_{j}^{n+1}=W_{j}^{n}+Q \cdot W_{j}^{n} .
$$

The nonlinear matrix operator $Q$ need not necessarily be the Lax-Wendroff operator appearing in Eq. (5). However, unless otherwise specified, $Q$ will be taken to mean the L-W operator. Let us next consider a mixed implicit-explicit finite-differencing scheme

$$
W_{j}^{n+1}=W_{j}^{n}+Q \cdot\left[\theta W_{j}^{n+1}+(1-\theta) W_{j}^{n}\right]
$$

where $0 \leqq \theta \leqq 1$. The case $\theta=0$ gives back the fully explicit form Eq. (12). The iterative scheme is based on (13) and the successive $s$ th iterate, denoted by $W^{n, s}$, satisfies the following recursive relation in $s$ :

$$
W_{j}^{n+1, s+1}=W_{j}^{n}+Q \cdot\left[\theta W_{j}^{n+1, s}+(1-\theta) W_{j}^{n}\right]
$$

where $s=0,1,2, \cdots, k-1$ and where we define $W_{j}^{n+1,0}=W_{j}{ }^{n}$. Note that the result of the first iteration $(s=0)$ is the same as the one obtained by solving the original difference equation (12). There will be $k$ iterations at each time step with $s$ increasing from 0 to $k-1$.

We now address ourselves to three questions:

(a) What is the stability criterion of the iterative scheme.

(b) What is the behavior of $W_{j}^{n+1, s}$ as $s \rightarrow \propto$.

(c) The numerical results for a small or moderate number of iterations, especially near shock waves.

Points (a) and (b) will be considered now. The numerical results will be described separately in Section 5 .

As usual the stability analysis of the scheme is carried out under the assumption that the matrix $A$ is locally constant. The case being examined is that where the zeroth iterate is the Lax-Wendroff finite-difference operator. Hence, for $A$ constant, we have, see Eq. (4) 


$$
\begin{aligned}
W_{j}^{n+1, s+1}= & W_{j}^{n}-\frac{\lambda}{2} A \cdot\left[\theta\left(W_{j+1}^{n+1, s}-W_{j-1}^{n+1, s}\right)+(1-\theta)\left(W_{j+1}^{n}-W_{j-1}^{n}\right)\right] \\
+ & \frac{\lambda^{2}}{2} A^{2} \cdot\left[\theta\left(W_{j+1}^{n+1, s}-2 W_{j}^{n+1, s}+W_{j-1}^{n+1, s}\right)\right. \\
& \left.\quad+(1-\theta)\left(W_{j+1}^{n}-2 W_{j}^{n}+W_{j-1}^{n}\right)\right] .
\end{aligned}
$$

Next define an operator $\Omega$ such that

$$
\Omega f_{j}^{n}=-\frac{\lambda}{2} \theta A \cdot\left(f_{j+1}^{n}-f_{j-1}^{n}\right)+\frac{\lambda^{2} \theta}{2} A^{2} \cdot\left(f_{j+1}^{n}-2 f_{j}^{n}+f_{j-1}^{n}\right) .
$$

Equation (15) is now written as

$$
W_{j}^{n+1, s+1}=(I+\mu \Omega) W_{j}^{n}+\Omega W_{j}^{n+1, s}
$$

where $\mu=(1-\theta) / \theta$; since we will consider $\frac{1}{2} \leqq \theta \leqq 1$ it follows that $1 \geqq \mu \geqq 0$. Since $W_{j}^{n+1,0}=W_{j}^{n}$ we immediately find

$$
\begin{aligned}
& W_{j}^{n+1,1}=[I+(1+\mu) \Omega] W_{j}^{n} \\
& W_{j}^{n+1,2}=\left[I+(1+\mu)\left(\Omega+\Omega^{2}\right)\right] W_{j}^{n} \\
& \cdot \cdot \\
& \cdot \\
& W_{j}^{n+1, k}=\left[I+(1+\mu) \sum_{r=1}^{k} \Omega^{r}\right] W_{j}^{n} .
\end{aligned}
$$

We follow von Neumann and let $W_{j}^{n}=R_{n} \exp \left[i \omega x_{j}\right]$ and substitute in Eq. (17) to find

$$
W_{j}^{n+1, k}=G \cdot W_{j}^{n}
$$

where the amplification matrix $G$ is given by

$$
G=I+(1+\mu) \sum_{r=1}^{k}(D+i B)^{r}
$$

with

$$
\begin{aligned}
D & =-2 \lambda^{2} \theta \cdot \sin ^{2} \phi \cdot A^{2} \\
B & =-\lambda \theta \cdot \sin 2 \phi \cdot A \\
\phi & =\omega \Delta x / 2 .
\end{aligned}
$$

If we denote the characteristic values of the matrices $D$ and $B$ by $d$ and $b$ respectively, then according to the spectral mapping theorem the corresponding eigenvalue of $G$, say $g$, is given by

$$
g=1+(1+\mu) \sum_{r=1}^{k}(d+i b)^{r}=-\mu+(1+\mu) \frac{1-(d+i b)^{k+1}}{1-(d+i b)} .
$$

We note here that it follows from the definition of $D$ and $B$ that

$$
d=-2 \lambda^{2} \theta \alpha^{2} \sin ^{2} \phi, \quad b=-\lambda \theta \alpha \cdot \sin 2 \phi,
$$

where $\alpha$ is a characteristic value of the matrix $A$. The von Neumann criterion for 
(linear) stability is that $|g|^{2}=g \cdot \bar{g} \leqq 1$. Carrying out the indicated manipulations this leads to:

$$
\begin{aligned}
H(\theta, \beta, y, k)=\left\{\theta^{2 k+1}(4 \beta y)^{k}(\beta y-y+1)^{k+1}+\theta^{k+1}(2 \beta y)^{k}(-1)^{k} S_{k+1}-\theta\right. \\
+(\beta y-y+1)(\theta-1)(2 \theta)^{k+1}(\beta y)^{k}(-1)^{k} S_{k} \\
+\theta(1-2 \theta)(\beta y-y+1)\} \leqq 0
\end{aligned}
$$

where

$$
\begin{aligned}
S_{k} & =\sum_{q=0}^{[k / 2]}\left(\begin{array}{c}
k \\
2 q
\end{array}\right)(-1)^{q}\left(\frac{1-y}{\beta y}\right)^{q}, \\
y & =\sin ^{2} \phi \\
\beta & =\lambda^{2} \alpha^{2} .
\end{aligned}
$$

Our task is to find the maximum $\beta$, for given natural number $k$, for a given $\frac{1}{2} \leqq \theta \leqq 1$, for all values of $y, 0 \leqq y \leqq 1$, such that the inequality (21) is still preserved. While we have not succeeded in solving (21) analytically (except for special values of $\theta$ and/or $y$ which served as a check on the numerical results), we carried out a numerical solution of $(21)$, from which the following conclusions are drawn:

(i) The solution of (21) may be expressed in the form: $\beta \leqq z^{2}(\theta, k) / 2 \theta$ which means that the desired stability criterion is of the form

$$
\frac{\Delta t}{\Delta x} \leqq \frac{z(\theta, k)}{(\max |\alpha|)(2 \theta)^{1 / 2}}
$$

where, it was found numerically, that $.995<z(\theta, k) \leqq 1$.

(ii) lor the special case $k=2 m, \frac{1}{2} \leqq \theta \leqq 1$, we find $z(\theta, 2 m)=1$.

(iii) $z\left(\frac{1}{2}, k\right)=1$.

(iv) $z(\theta, \infty)=1$.

In view of the above we decided to use a single stability criterion for all the various cases of different degree of implicity $(\theta)$ and different number of iterations $(k)$, namely

$$
\frac{\Delta t}{\Delta x}=\frac{0.95}{(\max |\alpha|)(2 \theta)^{1 / 2}}
$$

where the numerical coefficient provides us with a margin of safety. Note that $\theta=\frac{1}{2}$ leads us back to the CFL stability criterion, while for $\theta=1$ we get the most stringent one which calls for time steps which are $30 \%$ smaller.

Next we consider the behavior of $W_{j}^{n+1, k}$ as $k \rightarrow \infty$. We should like to find out under what conditions will $W_{j}^{n+1, k}$ approach the exact implicit solution. The analysis is carried out here also for $A$ constant. Equation (15) may be written as follows:

$$
\begin{aligned}
W_{j}^{n+1, s+1}= & W_{j}^{n}-\frac{\lambda}{2} A \cdot\left[W_{j+1}^{n+\theta, s}-W_{j-1}^{n+\theta, s}\right] \\
& +\frac{\lambda^{2}}{2} A^{2} \cdot\left[W_{j+1}^{n+\theta, s}-2 W_{j}^{n+\theta, s}+W_{j-1}^{n+\theta, s}\right]
\end{aligned}
$$

where 


$$
W_{j}^{n+\theta, s}=\theta W_{j}^{n+1, s}+(1-\theta) W_{j}^{n} .
$$

Now subtract from (24) the implicit equation which is of the same form except that instead of $W_{j}^{n+\theta, s}$ we have $\theta W_{j}^{n+1}+(1-\theta) W_{j}{ }^{n}$. With $e_{j}^{s}=W_{j}{ }^{n+1, s}-W_{j}{ }^{n+1}$ the resulting equation is:

$$
e_{j}^{s+1}=-\frac{\lambda}{2} A \cdot \theta\left(e_{j+1}^{s}-e_{j-1}^{s}\right)+\frac{\lambda^{2}}{2} A^{2} \cdot \theta\left(e_{j+1}^{s}-2 e_{j}^{s}+e_{j-1}^{s}\right) .
$$

If we use the same Fourier-type analysis as for the stability study i.e. substitute $e_{j}{ }^{k}=E_{s} \exp \left[i \omega x_{j}\right]$, we obtain after some manipulations

$$
e_{j}{ }^{s+1}=L e_{j}{ }^{s}=L^{2} e_{j}{ }^{s-1}=\cdots=L^{s+1} e_{j}{ }^{(0)}=L^{s+1}\left(W_{j}^{n}-W_{j}{ }^{n+1}\right)
$$

where the matrix operator $L$ is

$$
L=-i \lambda \cdot \theta \sin 2 \phi \cdot A-2 \lambda^{2} \theta \sin ^{2} \phi \cdot A^{2} .
$$

The characteristic value, $l$, of $L$ is given by the spectral mapping theorem

$$
l=-2 \lambda \theta \alpha \sin \phi(i \cos \phi+\lambda \alpha \sin \phi)
$$

and thus

$$
|l|^{2}=l \cdot \bar{l}=4 \beta y \theta^{2}(1-y+\beta y) \leqq(2 \beta \theta)^{2} \quad\left(\text { for } 0 \leqq y \leqq 1 ; \beta \geqq \frac{1}{2}\right)
$$

from (2i) we find that $e_{j}^{s+1} \rightarrow_{s \rightarrow \infty} 0$ only if $\max |l|<1$. Equation (26) shows that this condition is fulfilled only if $\beta<1 / 2 \theta$, or $\Delta t / \Delta x<1 /(\max |\alpha|)(2 \theta)^{1 / 2}$. Since our computational stability criterion, Eq. (23), meets this requirement the successive iterates approach the implicit solution. It can be shown that for $\theta=1$ the above criterion is still valid.

\section{Numerical Results. ${ }^{* *}$}

5.1. The Treatment of Equations of First-Order Accuracy. Before proceeding to present the results based on the scheme delineated above we make a short detour in order to describe the application of the iterative method to the first-order accuracy difference equations. This means we consider only the first two terms on the righthand side of Eq. (4). The resulting algorithm for this case is then

$$
W_{j}^{n+1, s+1}=W_{j}^{n}-\frac{\lambda}{2}\left[F_{j+1}^{n+\theta, s}-F_{j-1}^{n+\theta, s}\right]
$$

where

$$
F_{j}{ }^{n+\theta, s}=\theta F_{j}{ }^{n+1, s}+(1-\theta) F_{j}{ }^{n} .
$$

When $\theta=\frac{1}{2}$ this reduces exactly to the case treated by Gary [1], [2]. We have repeated his calculations for the case of weak shock wave $\left(p_{1} / p_{0}=1.4\right)$ and obtained the same result as reported by him. When, however, the same calculation (i. e. with $\left.\theta=\frac{1}{2}\right)$ is carried out even for a moderate shock strength $\left(p_{1} / p_{0}=4\right)$ the post shock oscillations are severe. This is seen in Fig. 1 . When the calculation was repeated for the same physical case (i.e. steady-plane shock wave, $p_{1} / p_{0}=4, \gamma=3$ ) but with

\footnotetext{
** In all of our cases the shock front moves from right to left, while the discrete Lagrangian coordinate runs from 1 to 201, i.e. $\Delta x=.005$. The initial time is $t=1.00$.
} 
$\theta=1$ the oscillations were reduced by a large amount even after one iteration - see Fig. 2.

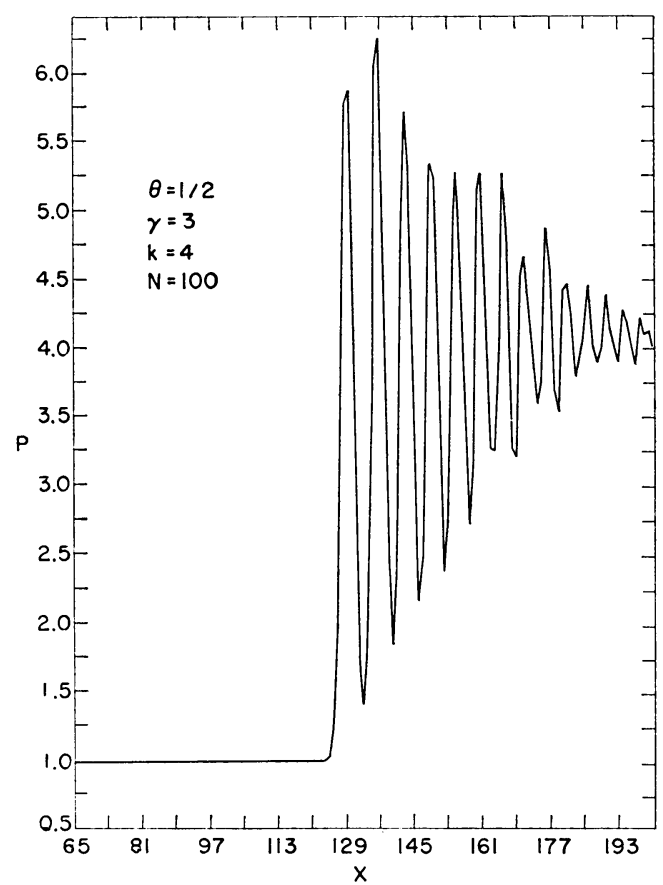

Figure 1. The pressure $p$ as function of the Lagrangian coordinate $x$, after $N=100$ time steps; computed by the method of [1]. Note the severe oscillations for this moderate strength shock wave.

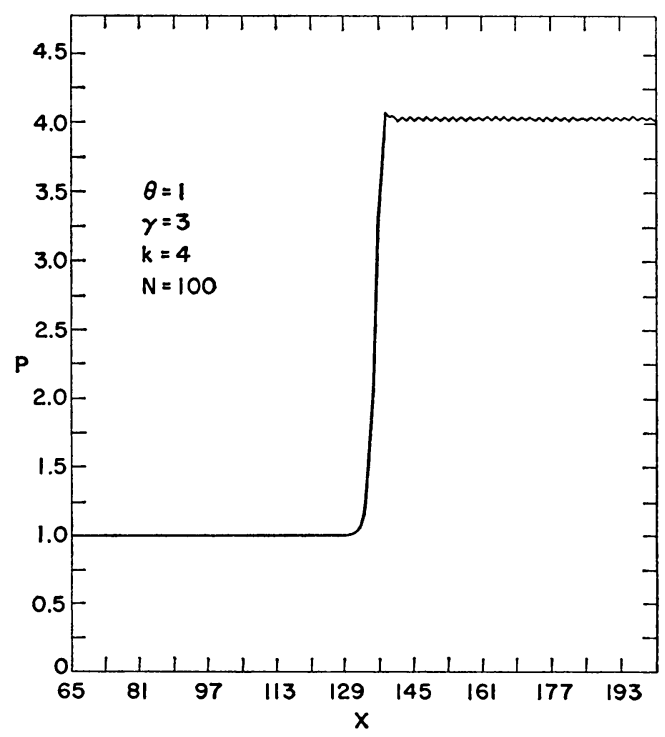

Figure 2. $p$ vs. $x$ as in Fig. 1 except that here $\theta=1$. 


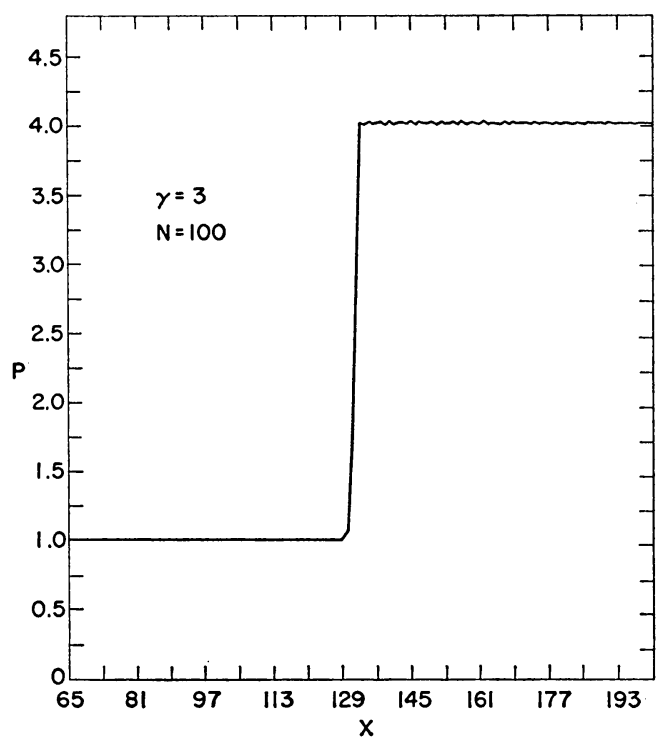

Figure 3. $p$ vs. $x$ computed by the method of Godunov.

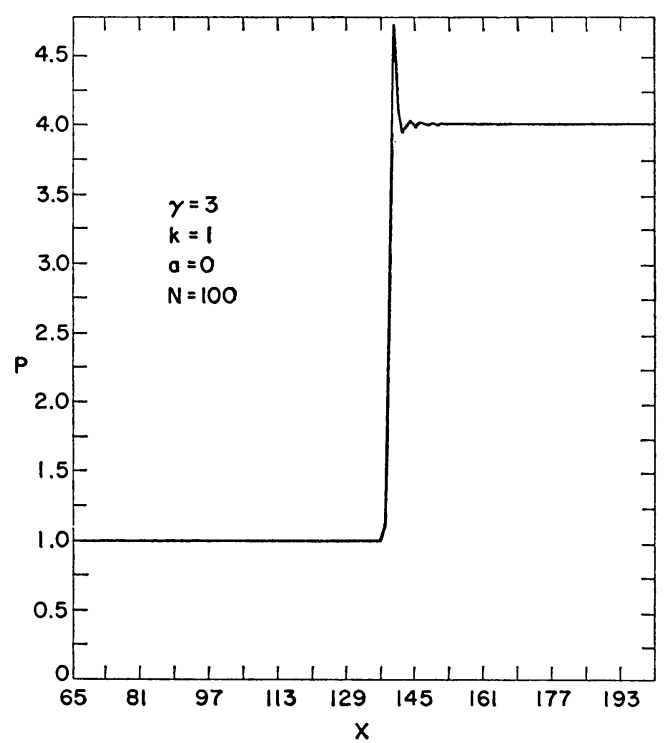

Figure 4. $p$ vs. $x$ computed by the Lax-Wendroff scheme. No artificial viscosity.

We shall now present a concise account of an analysis that might explain the "success" of the iterative method at $\theta=1$ and its failure at $\theta=\frac{1}{2}$. If one carries out the stability analysis as is done in Section 4, but for the system given by Eq. (27) rather than by Eq. (15), one finds two expressions for the magnitude of the characteristic value of the amplification matrix, depending on whether the number of iterations, $k$, is odd or even. 


$$
\begin{gathered}
|g|^{2}=\frac{1+\mu^{2} b^{2}}{1+b^{2}}+2(1+\mu)(-1)^{m} b^{2 m+2}\left[\frac{\left.1+\frac{1}{2} \frac{1+\mu)(-1)^{m} b^{2 m+2}}{1+b^{2}}\right]}{(k=2 m+1),}\right. \\
|g|^{2}=\frac{1+\mu^{2} b^{2}}{1+b^{2}}+2(1+\mu)(-1)^{m+1} b^{2 m+2}\left[\frac{\mu+\frac{1}{2}(1+\mu)(-1)^{m+1} b^{2 m}}{1+b^{2}}\right] \\
(k=2 m),
\end{gathered}
$$

where, as before, $\mu=(1-\theta) / \theta$ and $b=-\lambda \theta \alpha \sin 2 \phi$. If $\theta=\frac{1}{2}, \mu=1$ and the above expressions reduce to Gary's results [1] with the consequence that for $k=3$, $4,7,8, \cdots$ the scheme is stable, provided $\Delta t / \Delta x<2 /(\max |\alpha|)$, and unstable otherwise. However, we claim that since in order to perform three iterations the machine must go through the second iteration first, the scheme will be effectively unstable at all times. Figure 1 confirms this since the calculations were carried through four iterations, presumably a "stable" number. On the other hand for $\theta=1, \mu=0$, Eqs. (28) and (29) reduce respectively to

$$
|g|^{2}=\frac{\left[1+(-1)^{m} b^{2 m+2}\right]^{2}}{1+b^{2}}, \quad(k=2 m+1)
$$

and

$$
|g|^{2}=\frac{1+b^{4 m+2}}{1+b^{2}}, \quad(k=2 m) .
$$

The stability requirement is $|g|^{2} \leqq 1$. For even number of iterations, $k=2 m$, it follows immediately that this implies $|b| \leqq 1$ and hence $\lambda \leqq 1 / \max |\alpha|$. For $k$ odd we get $\lambda \leqq \delta / \max |\alpha|$ where $1 \leqq \delta<4 / 3$ for $k=4 r+3$ and $3 / 4<\delta<1$ if $k=4 r+1$. In any case it has just been demonstrated that for $\theta=1$ the scheme is stable for any number of iterations in contradistinction to the $\theta=\frac{1}{2}$ case. This may explain the small amplitude of the oscillations in Fig. 2. To provide a yard-stick for the "quality" of the scheme in the stable case $(\theta=1)$ we computed the same shock using a method due to Godunov [4] which is supposed to yield smooth profiles. As seen from Fig. 3, the pressure profile looks qualitatively the same as that in Fig. 2. The proof of the convergence of the iteration to the implicit solution in the case of general $\theta$ follows exactly the same lines as above and yields the criterion $\lambda \leqq 1 /(\max |\alpha|) \theta$.

5.2. Iterating the La. $x$-Wendroff Difference Scheme. We have solved numerically the L-W difference equations, with and without artificial viscosity-Eqs. (6) and (5) respectively. This was done for a polytropic gas without iterations $(k=1)$ and with iterations $(k \geqq 2)$, for many different values of the parameters $p_{1} / p_{0}, \gamma$, and $\theta$.

Consider the typical example of a plane steady shock wave with pressure ratio of 4 . Figures 4 to 7 show, respectively, the cases when the calculations were made (i) with no iterations $(k=1)$ and no artificial viscosity $(a=0)$, (ii) no iterations but with artificial viscosity $(a=2)$, (iii) one iteration $(k=2)$ and no artificial viscosity, and finally (iv) two iterations $(k=3)$ and no artificial viscosity. The plots show the pressure profiles rather than the velocity because we found the pressure to be more sensitive to perturbations. It is seen that the iterations are much 
more effective than the artificial viscosity in removing the oscillations. Notice that one iteration alone $(k=2)$ does most of the job and for $k=3$ the profile is very similar although the shock front is somewhat steeper. We also ran a twenty-iterations case and found that to the scale of the plot the results were indistinguishable from that of $k=3$. Another observation is that for different times the profiles were the same.

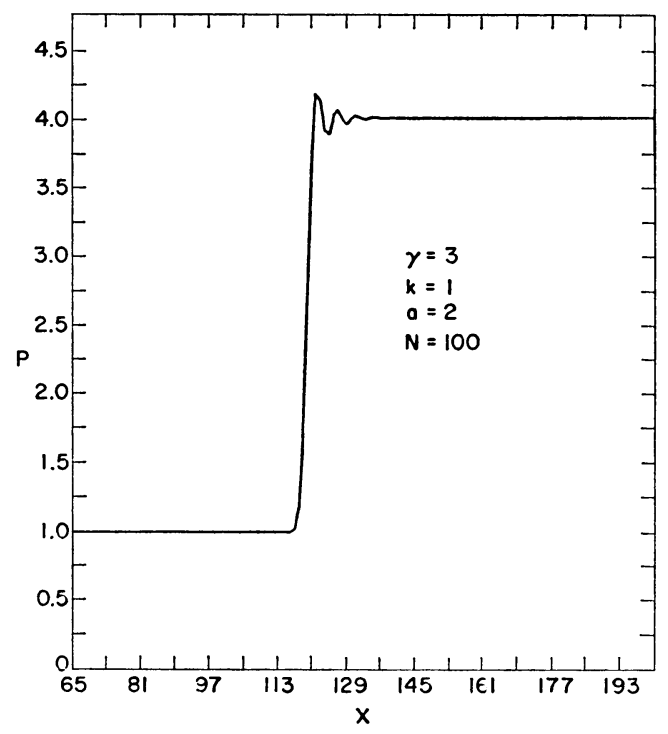

Figure 5. $p$ vs. $x$ computed as in Fig. 4 but with artificial viscosity.

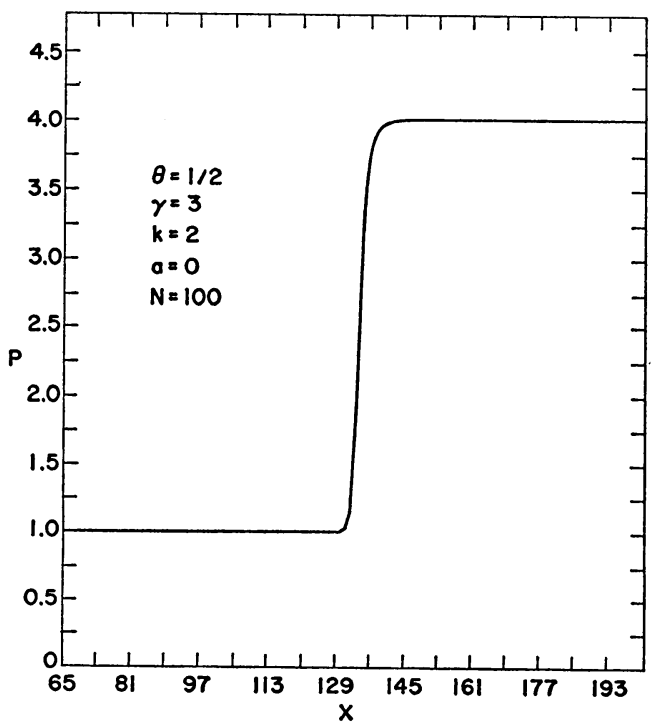

FIGURE 6. $p$ vs. $x$ computed iteratively with one iteration $(k=2)$. 


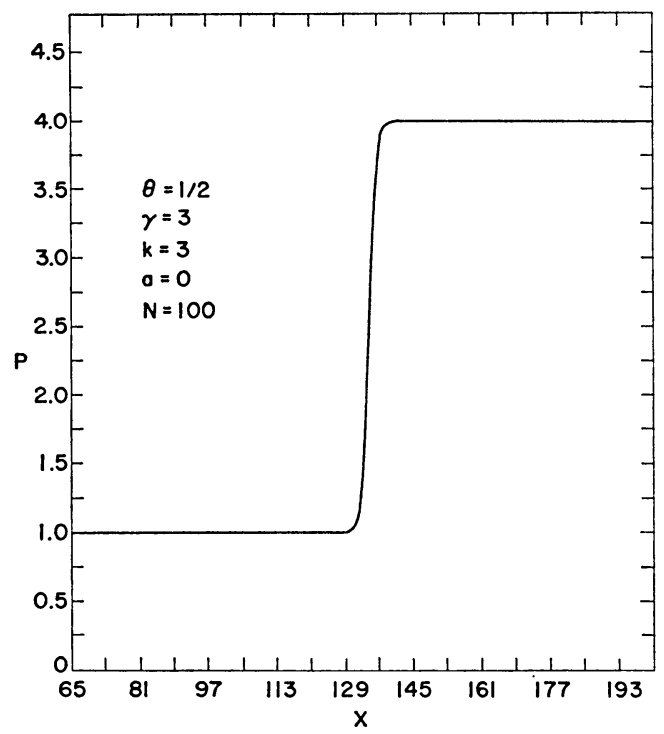

Figure 7. $p$ vs. $x$ with two iterations $(k=3)$.

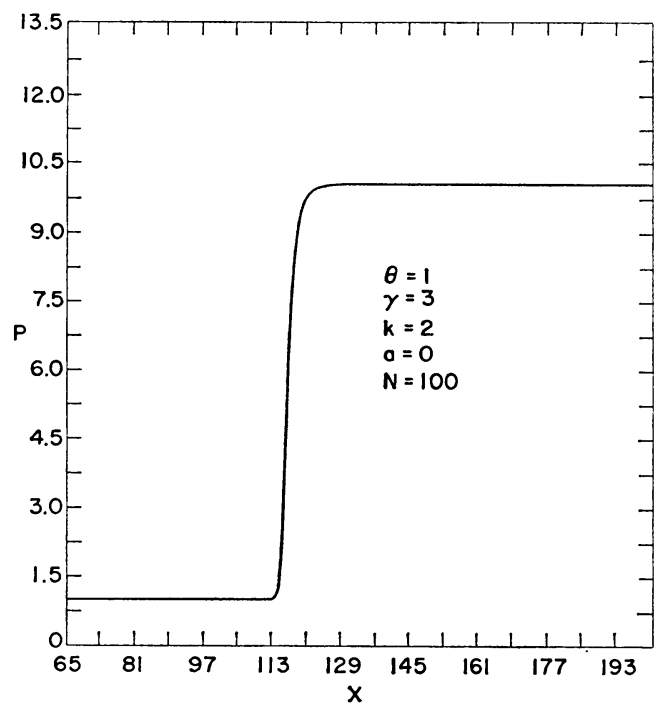

FIGURE 8. $p$ vs. $x$ computed iteratively for a strong shock wave $\left(p_{1} / p_{0}=10\right)$.

It occurred to us that the combination of a high pressure ratio and low $\gamma$ will present the most severe test to the iterative method. This is so because at high pressure ratios the specific volume ratio $v_{0} / v_{1}$ approaches its asymptotic value of $(\gamma+1) /(\gamma-1)$. That the high pressure by itself does not cause any problems one can see from Figs. $8,8 \mathrm{a}$ and $8 \mathrm{~b}$, where $p_{1} / p_{0}=10, \gamma=3,2$ and 1.4 respectively and after one iteration $(k=2)$ the profile is monotonic. Similarly we see from Fig. $8 \mathrm{c}$ that low $\gamma\left(\gamma=1.2 ; p_{1} / p_{0}=4\right)$ by itself also does not present any difficulties. We 
therefore tested the case of plane steady shock with $p_{1} / p_{0}=10$ and $\gamma=1.2$. Figures 9 and 10 present respectively the results for the no iterations case without and with artificial viscosity. It is seen that the overshoot for $k=1, a=0$ is much worse than for $p_{1} / p_{0}=4$ and that the artificial viscosity brings it down considerably. We found out that with iterations the results improved but that the scheme with $a>0$ worked better and we show the results in Fig. 11 for two iterations with $a=3 / 2$. While the profile is not monotonic, the overshoot is very small. For comparison we ran the same test case with the Godunov method-see Fig. 12. Clearly

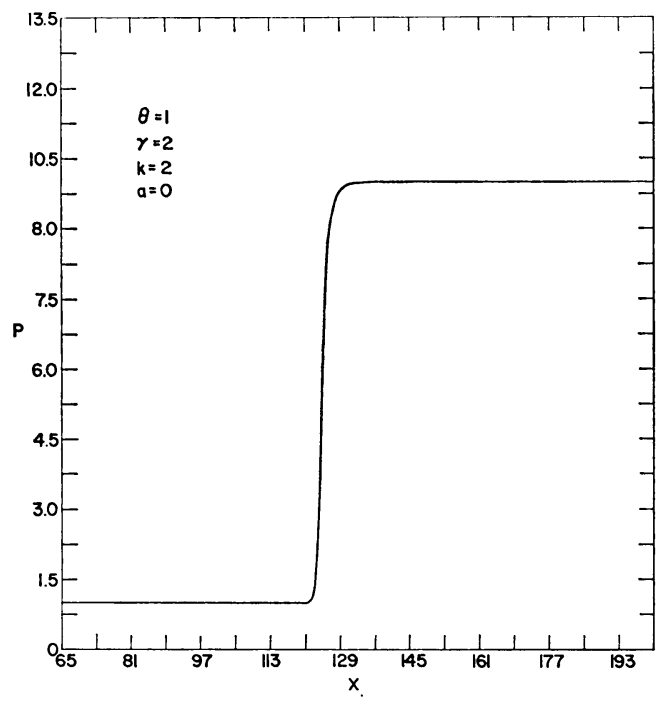

Figure 8 a) $p$ vs. $x$ as in Fig. 8 but for $\gamma=2$.

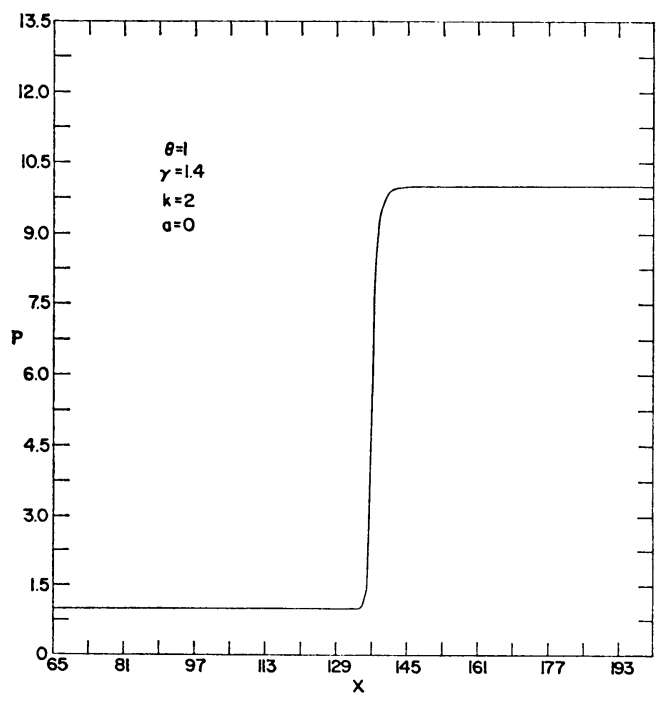

Figure 8 b) $p$ vs. $x$ as in Fig. 8 but for $\gamma=1.4$. 


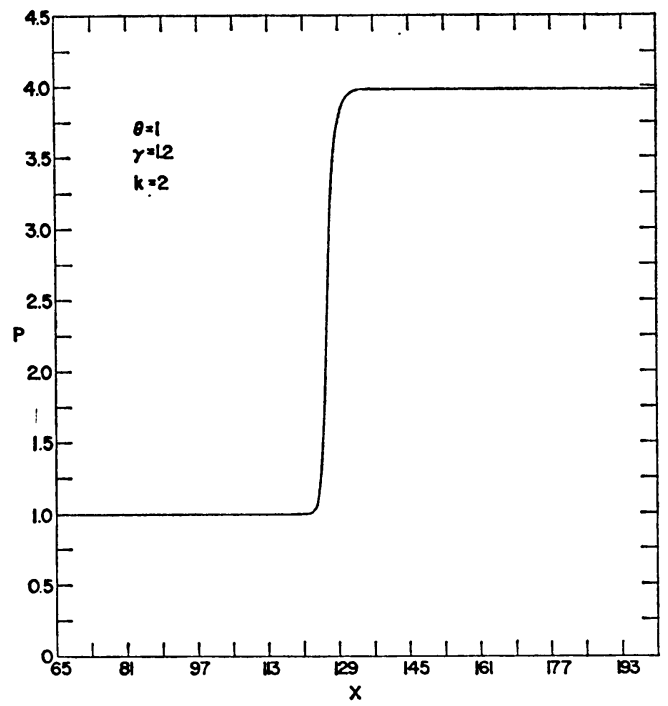

Figure 8 c) $p$ vs. $x$ as in Fig. 6 but for $\gamma=1.2$ and $\theta=1$.

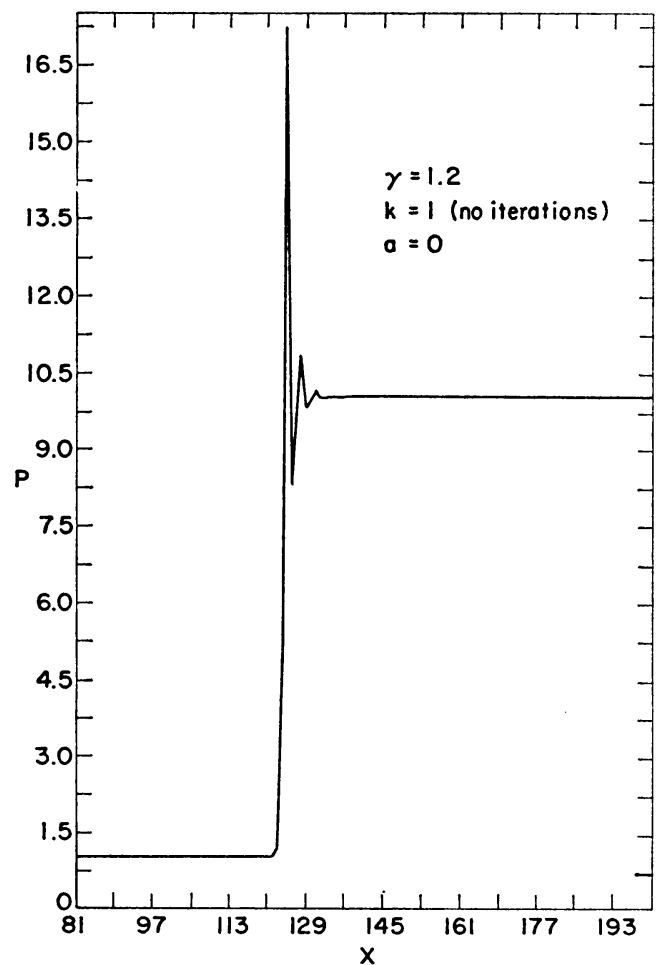

Figure 9. $p$ vs. $x$ for strong shock and small $\gamma$ - computed by the Lax-Wendroff scheme without artificial viscosity.

the iterative scheme gives a "cleaner" profile. All of the high pressure runs shown here were computed with $\theta=1$ which gives smoother results than $\theta=\frac{1}{2}$. In this sense the scheme with $\theta=1$ is more dissipative. 
In the course of these test cases and many other ones of low $\gamma$ and high $p_{1} / p_{0}$ we have encountered an interesting numerical behaviour which we would like to report. With no artificial viscosity the profile computed with one iteration $(k=2)$ has no overshoot. One more iteration "spoils" the profile. As more iterations are taken the profile becomes smooth again. However, for the case $\gamma=1.2, p_{1} / p_{0}=10$ it takes several tens of iterations to obtain results which are as good as those obtained with one iteration only. This phenomenon is noticeable, we stress again, only for the combination of low $\gamma$, and strong shock $\left(\gamma<1.4, p_{1} / p_{0} \geqq 10\right)$. Thus it seems that for all values of $\gamma$ and $p_{1} / p_{0}$ good results are obtained with one iteration only $(k=2)$.

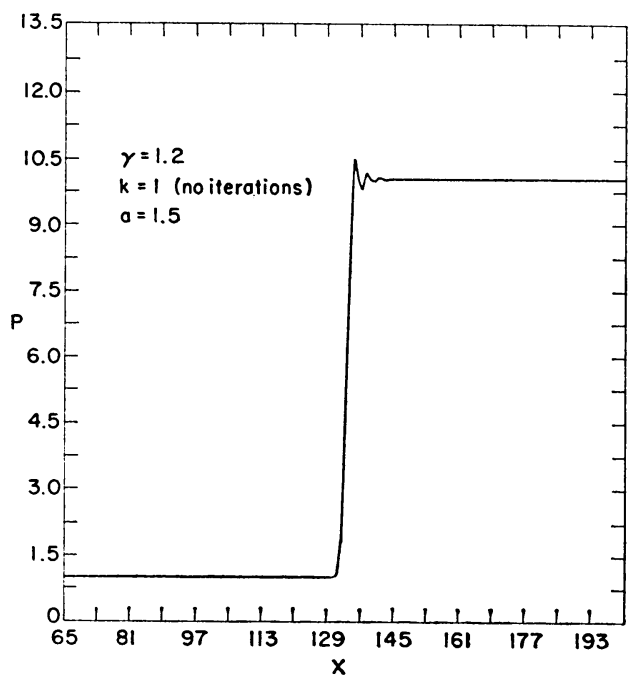

FIgURe 10. $p$ vs. $x$ as in Fig. 9 but with artificial viscosity.

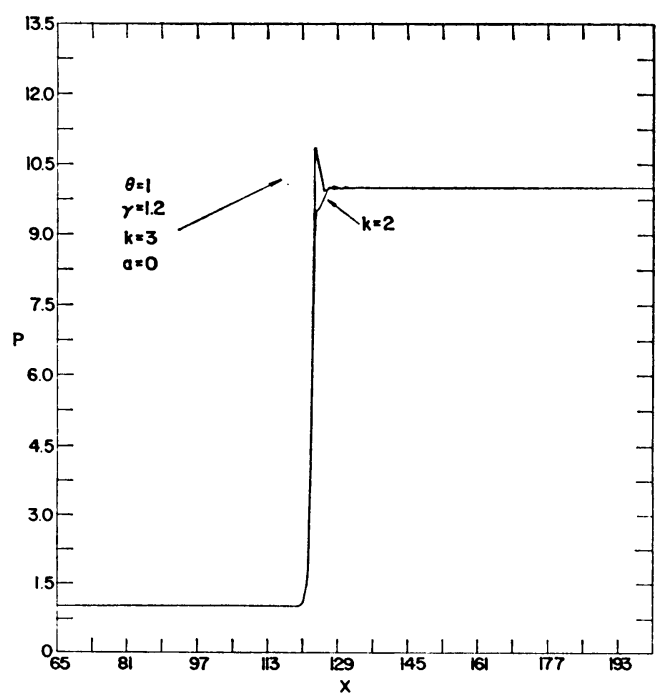

Figure 10 a) $p$ vs. $x$ as in Fig. 9 but with iterations. 


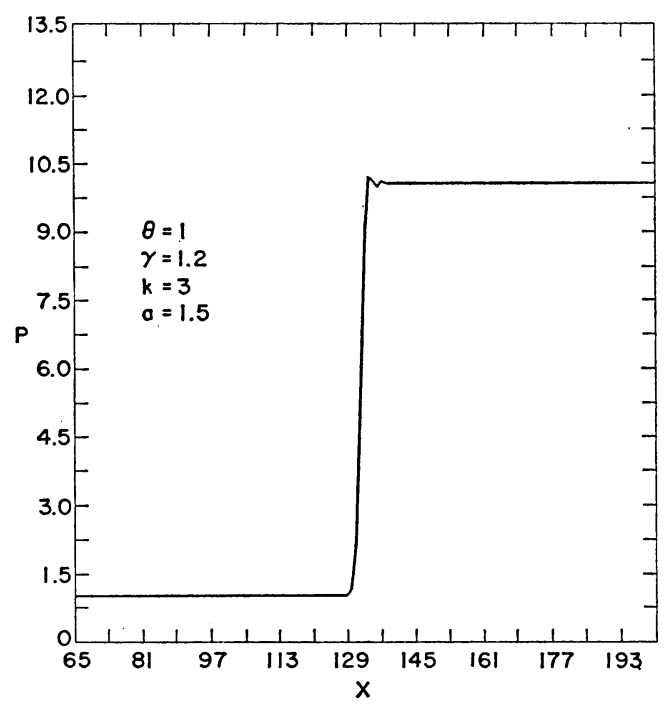

Figure 11. $p$ vs. $x$ as in Fig. 10, computed iteratively.

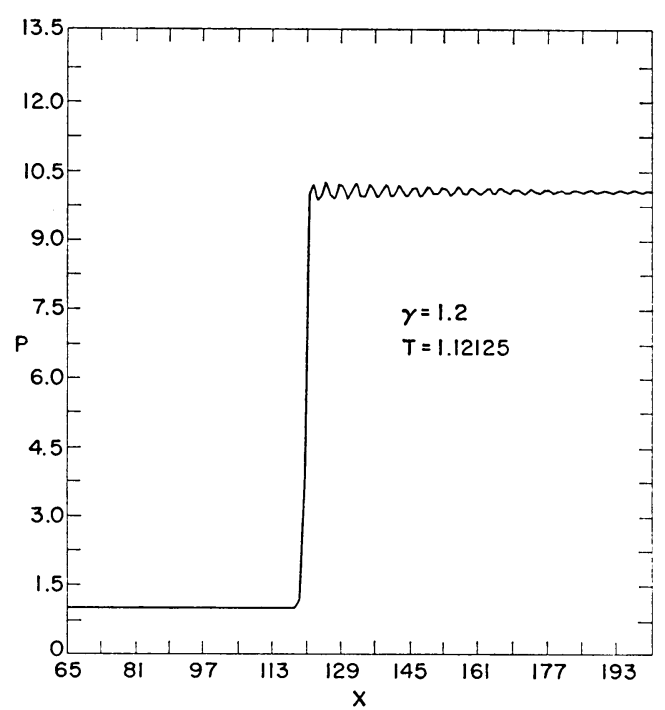

FIGLRE 12. $p$ vs. $x$ for strong shock and small $\gamma$ - computed by the method of Godunov.

Finally we decided to test the method in a nonsteady nonhomogenous case. The problem chosen is that of detonation wave $(\gamma=3)$ reflecting off a rigid wall as a hydrodynamic shock which moves into a moving nonhomogeneous region (the Taylor wave region-see references [6], [7] and [8]). Again the resulting profiles are monotonic-as shown in Fig. 13 in which the advancing wave is shown at several instances.

To summarize - the iterative scheme operating on the Lax-Wendroff difference equations gives very good results (monotonic profiles) for all the cases tested 


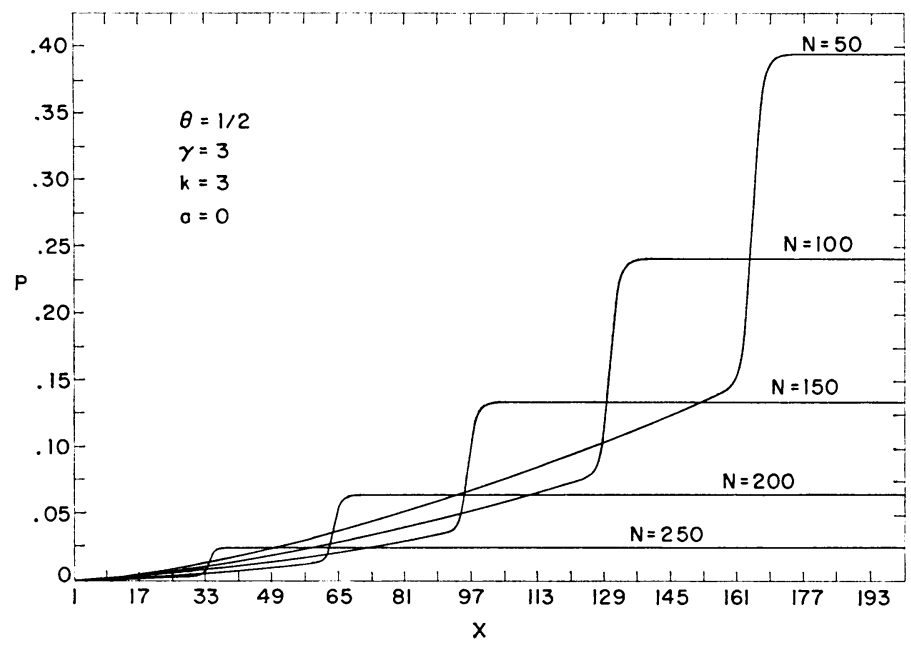

FIGLre 13. Shock wave reflecting off a wall as a result of a detonation wave impact. The pressure $p$ vs. $x$ at various times.

stationary and nonstationary, except when we had a confluence of a high pressure ratio $\left(p_{1} / p_{0}=10\right)$ and a low polytropic exponent $(\gamma=1.2)$. Even then the results are a big improvement over the computation without iterations.

Department of Applied Mathematics

Tel-Aviv University

Tel-Aviv, Israel

1. J. GARY, "On certain finite difference schemes for hyperbolic systems," Math. Comp., v. 18, 1964, pp. 1-18. IIR 28 \#1776.

2. J. Gary, On Certain Finite Difference Schemes for the Equations of Hydrodynamics, AEC leport, NYO-9188, 1962.

3. P. LAX \& B. Wendroff, "Systems of conservation laws," Comm. Pure Appl. Math., v. 13, 1960, pp. 217-237. MR 22 \#11523.

4. R. Richtmyki \& K. MonTon, Difference Methods for Initial Value Problems, Interscience Tracts in Pure and Appl. Math., no. 4, Interscience, New York, 1967. MR 36 \#3515.

5. E. Rubin \& S. Burstein, "IDifference methods for the inviscid and viscous equations of a compressible gas," J. Computational Phys., v. 2, 1967.

6. G. I. TAYLOR, "The dynamics of combustion products behind plane and spherical detonation fronts in explosives," Proc. Roy. Soc. London Ser. A., v. 200, 1950.

7. I. B. Zlildovich \& K. P. Stany ukovich, "On the reflection of a plane detonation wave," Dokl. A kad. Nauk SSSR, v. 5i), 1947. (Russian.)

8. S. ABARBANEL, E. Fishir \& G. ZWAs, "Trajectory of a rebounding detonation wave," AI.1AJ., v. ร), 1967.

9. A. R. Gourlay \& J. LL. Morris, "Finite-difference methods for nonlinear hyperbolic systems," Math. Comp., v. 22, 1968, pp. 28--39. MR $36 \# 6163$. 